Annals of Pure and Applied Mathematics

Vol. 16, No. 1, 2018, 37-46

ISSN: 2279-087X (P), 2279-0888(online)

Published on 1 January 2018

www.researchmathsci.org

DOI: http://dx.doi.org/10.22457/apam.v16n1a5

Annals of

Pure and Applied

Mathematics

\title{
Some Strong Connectivity Concepts in Weighted Graphs
}

\section{Darshan Lal M Nair ${ }^{1}$ and Sunil Mathew ${ }^{2}$}

Department of Mathematics

National Institute of Technology Calicut, India.

${ }^{1}$ Corresponding author. email: darshanlal.nair@gmail.com

${ }^{2}$ email: sm@nitc.ac.in

Received 5 November 2017; accepted 3 December 2017

\begin{abstract}
In this article, some new strong connectivity concepts in weighted graphs are studied. In many applications related with weighted graph networks, some connections do not contribute much to the network and hence some weak edges in the corresponding model can be ignored. Some new strong parameters namely strong independence number and strong covering number of a weighted graph are introduced and their relations are discussed.
\end{abstract}

Keywords: Strong independent set, strong covering set, strong independence number, strong covering number, strong matching.

AMS Mathematics Subject Classification (2010): $05 \mathrm{C} 72$

\section{Introduction}

Weighted graph theory, considered as added wings of graph theory, is flying high now as a part of applied Mathematics, as it gained its importance in various fields like interconnection networks, information theory, database theory etc. Intersection graphs introduced by $\mathrm{Pal}$ [9] also exhibit an important relation between communication system and graph theory. Connectivity is one main concept underlying applications of weighted graphs and graphs. Minimum and maximum spanning tree problems, strong cycles and paths, all play major roles in related applications. Several authors including Bondy and Fan[2, 3], Bondy et al.[1] and Mathew and Sunitha [13, 14, 15] had put forward several connectivity concepts in weighted graphs inspired from the spark given by Dirac [6] and Grotschel [8]. Similar definitions in fuzzy graph are also extracted by Sunitha and Mathew [16]. The concepts introduced by these authors include partial cutvertices, partial bridges and partial blocks. Mathew and Sunitha have characterized partial cutvertices and partial bridges recently [12].

In this article we intend to throw light on some new adjacency properties of weighted graphs. The inspiration behind introducing these properties is that, the reduction of flow between some pairs of vertices is something which occurs more frequently than the total disconnection in the flow or disconnection of the entire network. Since weighted graphs is an extension of graphs, the concepts introduced here are also extensions of the classic connectivity concepts. 


\section{Darshan Lal M Nair and Sunil Mathew}

\section{Basic concepts}

A weighted graph $G$ is a graph in which every edge $e$ is assigned a non-negative number $w(e)$, called the weight of $e$. The set of all the neighbours of a vertex $v$ in $G$ is denoted by $N_{G}(v)$ or simply $N(v)$, and its cardinality by $d_{G}(v)$ or $d(v)$ [5]. The weighted degree of $v$ is defined as $w d_{G}(v)=\Sigma_{x \in N(v)} w(v, x)$. When no confusion occurs, we denote $w d_{G}(v)$ by $w d(v)$. The weight of a cycle is defined as the sum of the weights of its edges. An unweighted graph can be regarded as a weighted graph in which every edge $e$ is assigned a weight one. Thus, in an unweighted graph, $w d(v)=d(v)$ for every vertex $v$, and the weight of a cycle is simply the length of the cycle. An optimal cycle is a cycle which has maximum weight. A path in a weighted graph $G$ (weighted path) is a sequence of vertices and edges with a weight assigned to each edge. A weighted graph $\mathrm{G}$ is connected, if every pair of vertices are connected by a weighted path. Two paths, say $P_{1}$ and $P_{2}$, are said to be edge disjoint if they do not have any common edge and disjoint if they do not share any common vertex. Two $u-v$ paths are said to be internally disjoint, if they have no common vertices other than $u$ and $v$. A vertex $v$ is said to be a cut vertex of $G$, if its removal from the graph disconnects the graph $G$ and an edge in $G$ is called a cut edge or a bridge if its removal disconnects $G$. A maximum spanning tree (MST) of a weighted graph $G$ is a spanning graph of $G$, which is a tree and the sum of weights of its edges, the largest among all such trees. Some more definitions are given below.

Apart from the above given basic definitions, there exists a few more concepts related to connectivity which play an important role in applications of weighted graphs. Next we present a few of them, which includes strength of connectedness between pair of vertices, different types of edges, etc. Strength of connectedness and strength of path do have their own significance in determining distinct capacities in different types of networks.

Let $G$ be a weighted graph. The strength of a path $P$ (respectively, strength of a cycle $C)$ [12] of $n$ edges $e_{i}$, for $1 \leq i \leq n$, denoted by $s(P)$ (respectively, $s(C)$ ), is equal to $s(P)=\min _{1 \leq i \leq n}\left\{w\left(e_{i}\right)\right\}$. The strength of connectedness of a pair of vertices $u, v \in V(G)$, denoted by $\operatorname{CONN}_{G}(u, v)[12]$, is defined as $\operatorname{CONN}_{G}(u, v)=$ $\operatorname{Max}\{\mathrm{s}(\mathrm{P}): \mathrm{P}$ is a $\mathrm{u}-\mathrm{v}$ path in $\mathrm{G}\}$.

Example 2.1. Let $G(V, E)$ be a weighted graph(Fig. 1) with $V=\{a, b, c, d\}$ and $E=\left\{e_{1}=(a, b), e_{2}=(b, c), e_{3}=(c, d), e_{4}=(d, a), e_{5}=(b, d), e_{6}=(a, c)\right\}$ with $w\left(e_{1}\right)=4, w\left(e_{2}\right)=5, w\left(e_{3}\right)=6, w\left(e_{4}\right)=2, w\left(e_{5}\right)=8, w\left(e_{6}\right)=3$.

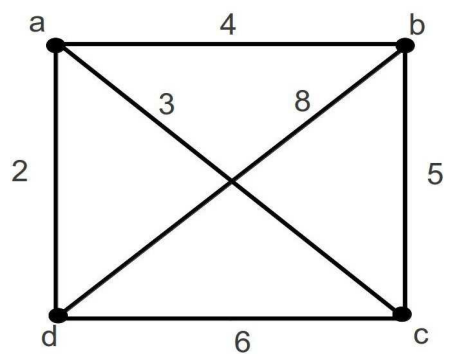

Figure 1: Strength of connectedness 
In this weighted graph (Fig. 1), $\operatorname{CONN}_{G}(a, b)=\max \{4,3,3,2,2\}=4$, $\operatorname{CONN}_{G}(b, c)=\max \{5,3,6,2,2\}=6, \operatorname{CONN}_{G}(b, d)=\max \{8,5,2,2,3\}=8$.

If $G$ is a weighted graph and $H$ a weighted subgraph of $G$, then for every pair of vertices $u, v \in V$, we have $\operatorname{CONN}_{H}(u, v) \leq \operatorname{CONN}_{G}(u, v)$. If $u$ and $v$ are in different components of $G$, then $\operatorname{CONN}_{G}(u, v)$ equals zero. A $u-v$ path in a weighted graph $G$ is called a strongest $u-v$ path[12] if $s(P)=\operatorname{CONN}_{G}(u, v)$. A vertex $w$ is called a partial cutvertex (p-cutvertex for short)[12] of $G$ if there exists a pair of vertices $u, v$ in $G$ such that $u \neq v \neq w$ and $\operatorname{CONN}_{G-w}(u, v)<\operatorname{CONN}_{G}(u, v)$. A connected weighted graph having no p-cutvertex is called a partial block (p-block for short)[12]. An edge $e=(u, v)$ is called a partial bridge (p-bridge for short) if $\operatorname{CONN}_{G-e}(u, v)<$ $\operatorname{CONN}_{G}(u, v)$. A p-bridge $e$ is said to be a partial bond (p-bond for short) if $\operatorname{CONN}_{G-e}(x, y)<\operatorname{CONN}_{G}(x, y)$ with at least one of $x$ or $y$ different from both $u$ and $v$ and is said to be a partial cutbond (p-cutbond for short) if both $x$ and $y$ are different from $u$ and $v$. An edge $e$ is said to be a weakest edge of a weighted graph $G$ if $w(e) \leq w\left(e^{\prime}\right)$ for any other edge $e^{\prime}$ of $G$.

An edge $e=(x, y)$ is strong if its weight is at least equal to the strength of connectedness between $x$ and $y$ in $G$ and edge $e=(x, y)$ is called $\alpha$-strong if $\operatorname{CONN}_{G-e}(x, y)<w(e), \beta$-strong if $\operatorname{CONN}_{G-e}(x, y)=w(e)$ and a $\delta$-edge if $\operatorname{CONN}_{G-e}(x, y)>w(e)$. A $\delta$-edge $e$ is called a $\delta^{*}$-edge if $e$ is not a weakest edge of $G$ [12]. A $u-v$ path $P$ in $G$ is called a strong $u-v$ path if all edges in $P$ are strong. In particular if all edges of $P$ are $\alpha$-strong, then $\mathrm{P}$ is called an $\alpha$-strong path and if all edges of $P$ are $\beta$-strong, then $P$ is called a $\beta$-strong path. A cycle $C$ in $G$ is called a strong cycle if all edges in $C$ are strong. An edge $(x, y)$ in a weighted graph $G$ is strong if and only if $w(e)=\operatorname{CONN}_{G}(x, y)$. An edge $e$ in a weighted graph $G$ is a partial bridge if and only if $e$ is $\alpha$-strong. If $x$ and $y$ are any two vertices in a connected weighted graph $G$, then there exists a strong path from $x$ to $y$.

A connected weighted graph $G=(V, E)$ is called a weighted partial tree (partial tree in short) [11] if $\mathrm{G}$ has a spanning subgraph $F=\left(V, E^{\prime}\right)$ which is a tree, where for all edges $(x, y)$ of $G$ which are not in $F$, we have $\operatorname{CONN}_{G}(x, y)>w(x, y)$. When the graph $G$ is not connected and if the above condition is satisfied by all components of $G$, then $G$ is called a partial forest.

Consider the following weighted graph (Fig. 2).

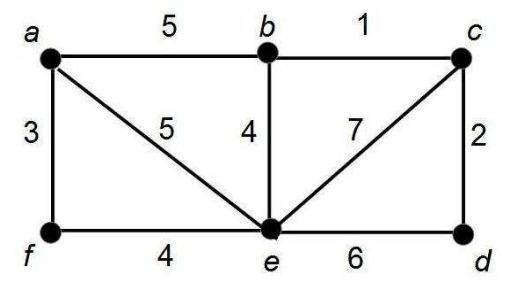

Figure 2: Partial tree

In this weighted graph (Fig. 2), by removing the edges $(a, f),(b, e),(b, c)$ and $(c, d)$ we will get the spanning tree $F$. Hence the above weighted graph is a partial tree. 
Darshan Lal M Nair and Sunil Mathew

Theorem 2.1. [11] A connected weighted graph $G$ is a partial tree if and only if in any cycle $C$ of $G$, there exists an edge $e=(x, y)$ such that $w(e)<\operatorname{CONN}_{G-e}(x, y)$, where $G-e$ is the subgraph of $G$ obtained by deleting the edge e from $G$.

Theorem 2.2. [11] If $G$ is a weighted partial tree and is not a tree, then there exists at least one edge $(u, v)$ for which $w(u, v)<\operatorname{CONN}_{G}(u, v)$.

Theorem 2.3. [11] If $G$ is a partial tree and $F$, the spanning tree in the definition, then the edges of $F$ are the partial bridges of $G$.

A totally weighted graph [10] is a weighted graph $G(V, E)$ with weight functions $\sigma: V \rightarrow R^{+}$and $\mu: E \rightarrow R^{+}$such that $\mu(x, y) \leq \sigma(x) \wedge \sigma(y)$ for any pair of vertices $x, y$ of $G$, where $\wedge$ denotes the minimum. A precisely weighted graph[10] is a totally weighted graph $G$ with weight functions $\sigma: V \rightarrow R^{+}$and $\mu: E \rightarrow R^{+}$such that $\mu(x, y)=\sigma(x) \wedge \sigma(y)$ for any pair of vertices $x, y$ of $G$, where $\wedge$ denotes the minimum. A precisely weighted graph has no $\delta$-edges. sessions.

We shall now discuss some new concepts on connectivity in the following

\section{Strong independent set and strong covering set}

The concepts of independent sets and covers do exist in graphs [7] as well as in semigraphs [17]. They can be easily extended to weighted graphs. Since they deal with adjacency of vertices, their extensions are similar.

Definition 3.1. Let $G=(V, E)$ be a weighted graph. A subset $S$ of $V$ is said to be a strong independent set if for any pair of vertices $u, v$ in $S,(u, v)$ is not a strong edge. A maximum strong independent set is a subset $S$ of $V$ such that $S$ is a strong independent set and for any other strong independent set $S^{\prime},\left|S^{\prime}\right| \leq|S|$. $|S|$, where $S$ is any maximum strong independent set, is called strong independence number of $G$, denoted by $\beta_{S}(G)$.

Definition 3.2. Let $G=(V, E)$ be a weighted graph. A subset $S$ of $V$ is said to be a strong covering set of $G$ if every strong edge of $G$ is incident with at least one vertex in $S$. A minimum strong covering set of $G$ is a subset $S$ of $V$ such that $S$ is a strong covering set and for any other strong covering set $S^{\prime}$ of $G,\left|S^{\prime}\right| \geq|S|$. $|S|$, where $S$ is any minimum strong covering set, is called strong covering number of $G$, denoted by $\alpha_{s}(G)$.

Example 3.1. Let $G(V, E)$ be a weighted graph(Fig. 3) with $V=\{p, q, r, s\}$ and $E=\left\{e_{1}=(p, q), e_{2}=(q, r), e_{3}=(r, s), e_{4}=(s, p), e_{5}=(q, s)\right\} \quad$ with $\quad w\left(e_{1}\right)=$ $2, w\left(e_{2}\right)=1, w\left(e_{3}\right)=5, w\left(e_{4}\right)=3, w\left(e_{5}\right)=4$. 
Some Strong Connectivity Concepts in Weighted Graphs

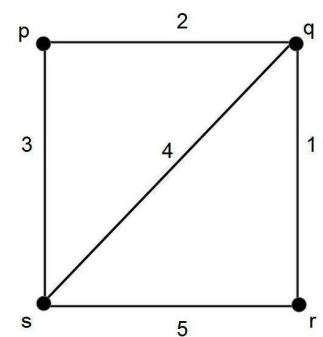

Figure 3: Strong independent set and strong covering set

In the above example all edges except $e_{1}$ and $e_{2}$ are strong. $\{p, q\},\{p, r\},\{q, r\}$ and $\{p, q, r\}$ are the strong independent sets. Hence the strong independence number is three. Since all strong edges in the above graph are incident with the vertex $s$, singleton set $\{s\}$ itself is a strong covering set and also it is the minimum strong covering set. Hence strong covering number of $G$ is one.

Note that if we consider the underlying graph of the above weighted graph, then its vertex independence number and vertex covering number are both equal to two. Hence it differs from the strong independence number and strong covering number of the same graph with weights given to its edges.

The following are few results in which we have found the strong covering number and strong independence number of different types of weighted graphs. The obvious proofs are omitted.

Theorem 3.1. Let $C$ be a strong cycle with $p$ vertices. Then,

1. $\beta_{s}(C)=\lfloor p / 2\rfloor$

2. $\alpha_{s}(C)=[p / 2\rceil$

Theorem 3.2. Let $G$ be a precisely weighted graph with $p$ vertices. Then,

1. $\beta_{s}(G)=1$

2. $\alpha_{S}(G)=p-1$

Proof: $G$ being a precisely weighted graph, is a complete graph with all edges being strong. Hence the strong independent sets are only the singleton sets.

Since $G$ is a complete graph with all edges being strong, minimum $p-1$ vertices are required to cover all the strong edges and hence $\alpha_{s}(G)=p-1$.

Theorem 3.3. In a strongly bipartite graph[4], each partition is a strong independent set as well as a strong covering set.

Proof: Let $G$ be a strongly bipartite graph. Let $V_{1}$ and $V_{2}$ be the 2 partitions in which the end vertices of strong edges lie. $G$ being strongly bipartite, no two vertices of $V_{1}$ and $V_{2}$ make a strong edge. Hence both $V_{1}$ and $V_{2}$ are strong independent sets.

Since every strong edge has one end vertex in $V_{1}$ and the other in $V_{2}$, taking either $V_{1}$ or $V_{2}$ will cover all the strong edges in $G$. Hence both $V_{1}$ and $V_{2}$ are strong covering sets of $G$.

Theorem 3.4. In a partial tree with more than two vertices, the set of pendant vertices of the unique maximum spanning tree in the definition of partial tree form a strong 


\section{Darshan Lal M Nair and Sunil Mathew}

independent set.

Proof: Let $G$ be a partial tree and let $F$ be the unique maximum spanning tree in the definition of $G$. Let $S$ be the set of pendant vertices of $F$ and hence no two vertices in $S$ are adjacent in $F$. Let $u, v$ be any two vertices in $S .(u, v)$ not being an edge in $F,(u, v)$ is not a strong edge of $G$ by the definition of partial tree. Hence $S$ is a strong independent set.

Theorem 3.5. For any connected weighted graph $G, \alpha_{s}(G)+\beta_{S}(G)=p$, where $p$ is total number of vertices in $G$.

Proof: Since there exists a strong path between any pair of vertices, there exists a strong edge adjacent to any vertex.

Let $\beta_{S}(G)$ be the strong independence number of $G$ and let $\alpha_{s}(G)$ be the strong covering number of $G$. Let $S$ be the maximum strong independent set such that $|S|=$ $\beta_{S}(G) . S$ being maximum strong independent set, no strong edge will have its both end vertices in $S$. Also each strong edge will have its one end vertex in $V \backslash S$. Hence $V \backslash S$ is a strong covering set of $G$. By the definition of $\alpha_{s}(G), \alpha_{S}(G) \leq|V \backslash S|=p-\beta_{S}(G)$. Hence we get

$$
\alpha_{s}(G)+\beta_{s}(G) \leq p
$$

Let $T$ be a minimal strong covering set such that $|T|=\alpha_{S}(G)$. $T$ being a strong covering set, no strong edge will have both its end vertices in $V \backslash T$. Hence $V \backslash T$ is a strong independent set. By the definition of $\beta_{s}(G), \beta_{s}(G) \geq|V \backslash T|=p-\alpha_{s}(G)$. Hence we get

$$
\alpha_{s}(G)+\beta_{s}(G) \geq p
$$

From equations (1) and (2), $\alpha_{s}(G)+\beta_{s}(G)=p$.

Consider Figure 3. In this weighted graph, strong independence number is three and strong covering number is one. Hence the sum equals four, which is the total number of vertices in the weighted graph.

\section{Strong edge independent set and strong edge covering set}

In this section we discuss the edge analogues of strong independent and covering sets. They can be considered as extensions of line independent sets and line covering sets.

Definition 4.1. Let $G(V, E)$ be a weighted graph. A collection $Q$ of strong edges is said to be a strong edge independent set if no two strong edges in $Q$ are adjacent. The strong edge independence number of a weighted graph $G$, denoted by $\beta_{s}^{\prime}(G)$, is defined as $\beta_{s}^{\prime}(G)=$ $\max \{|Q|: Q$ is any strong edge independent set $\}$.

Definition 4.2. A collection of strong edges $R$ is said to be a strong edge covering set of weighted graph $G$ if every vertex of $G$ is incident with at least one edge of $R$. The strong edge covering number of a weighted graph $G$, denoted by $\alpha_{s}^{\prime}(G)$, is defined as $\alpha_{s}^{\prime}(G)=$ $\min \{|R|: R$ is any strong edge covering set $\}$.

Example 4.1. Let $G(V, E)$ be a weighted graph with $V=\{a, b, c, d, e\}$ and $E=\left\{e_{1}=\right.$ $\left.(a, b), e_{2}=(b, c), e_{3}=(c, d), e_{4}=(d, e), e_{5}=(e, a), e_{6}=(e, b)\right\}$ with $w\left(e_{1}\right)=$ 
Some Strong Connectivity Concepts in Weighted Graphs

$5, w\left(e_{2}\right)=8, w\left(e_{3}\right)=4, w\left(e_{4}\right)=7, w\left(e_{5}\right)=3, w\left(e_{6}\right)=6$.

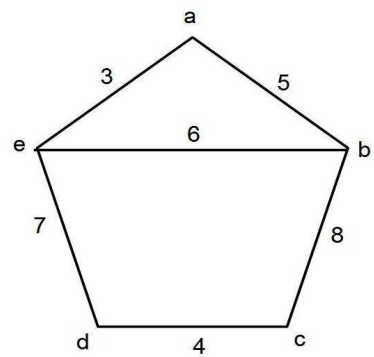

Figure 4: Strong edge independent set and strong edge covering set

Consider the above weighted graph. The strong edges in this weighted graph are $e_{1}, e_{2}, e_{4}$ and $e_{6}$. The strong edge independent sets in this particular weighted graph are $\left\{e_{6}\right\},\left\{e_{1}, e_{4}\right\}$ and $\left\{e_{2}, e_{4}\right\}$. Hence the strong edge independence number of $\mathrm{G}$ is two. Since there is only one strong edge incident with the vertices $a, c$ and $d$, all these three edges need to be included in any strong edge covering set and in fact, that is the minimum number of strong edges which will cover all the vertices. Hence the strong edge covering number of this weighted graph is three.

Theorem 4.1. For a strong cycle $C$ with $p$ vertices, we have:-

1. $\beta_{s}^{\prime}(C)=\lfloor p / 2\rfloor$

2. $\alpha_{s}^{\prime}(C)=\lceil p / 2\rceil$

Theorem 4.2. For a precisely weighted graph $G$ with $p$ vertices, we have:-

1. $\beta_{s}^{\prime}(G)=\lfloor p / 2\rfloor$

2. $\alpha_{s}^{\prime}(C)=\lceil p / 2\rceil$

Proof: A precisely weighted graph is a complete graph with all edges being strong. Hence the maximum number of mutually non-adjacent strong edges is equal to the maximum number of distinct pair of vertices which can be chosen from $p$ vertices at a time. Hence, $\beta_{s}^{\prime}(G)=\lfloor p / 2\rfloor$.

Similarly, to cover all the vertices in a precisely weighted graph, we require a minimum of either $p / 2$ strong edges if $p$ is even or $p / 2+1$ strong edges if $p$ is odd. Hence, $\alpha_{s}^{\prime}(C)=\lceil p / 2\rceil$.

Theorem 4.3. For any weighted graph $G, \alpha_{s}^{\prime}(G)+\beta_{s}^{\prime}(G)=p$, where $p$ is the total number of vertices in $G$.

Proof: Let $R$ be a strong edge covering set such that $|R|=\alpha_{s}^{\prime}(G)$. Let $R^{\prime}$ be the subgraph induced by the strong edges in $R$. Then $R^{\prime}$ cannot contain a path of length three (as all the four vertices in the path can be covered by just two edges and $R$ is a minimum strong edge covering set). Thus $R^{\prime}$ is a union of, say, $k$ star graphs. Each star graph being a tree we have

$$
\alpha_{s}^{\prime}(G)+k=p
$$

If we choose one edge from each component, then those $k$ edges form a strong 


\section{Darshan Lal M Nair and Sunil Mathew}

edge independent set. $\beta_{s}^{\prime}(G)$ being the strong edge independence number, $k \leq \beta_{s}^{\prime}(G)$. Hence from equation (3), we get

$$
\alpha_{s}^{\prime}(G)+\beta_{s}^{\prime}(G) \geq p
$$

To prove the other inequality, let $Q$ be a strong edge independent set such that $|Q|=\beta_{s}^{\prime}(G)$. Hence $Q$ contains $2 \beta_{s}^{\prime}(G)$ vertices. Consider the $p-2 \beta_{s}^{\prime}(G)$ vertices which are not incident with any strong edge in $Q$. Since there exists a strong edge incident to any vertex in $G$, we could choose $p-2 \beta_{s}^{\prime}(G)$ strong edges which are distinct from strong edges in $Q$. These edges together with edges in $Q$ form a strong edge covering set of $G$. $\alpha_{s}^{\prime}(G)$ being the minimum of cardinality of all such sets, we get $p-2 \beta_{s}^{\prime}(G)+$ $\beta_{s}^{\prime}(G) \geq \alpha_{s}^{\prime}(G)$. Hence

$$
\alpha_{s}^{\prime}(G)+\beta_{s}^{\prime}(G) \leq p
$$

Hence from equations (4) and (5), we get $\alpha_{s}^{\prime}(G)+\beta_{s}^{\prime}(G)=p$.

Consider the weighted graph in Figure 4. The strong edge independence number is two and the strong edge covering number is three. Hence the sum equals five, the total number of vertices in the weighted graph.

\section{Strong matching}

A collection of independent edges is considered as a matching[7]. It establishes a relation between the adjacent pairs of vertices or in fact, non adjacent edges. An analogous definition which relates the end vertices of a strong edge is being defined in this section. We make use of strong independent edges in weighted graphs for the analogous definition.

Definition 5.1. Let $G$ be a weighted graph. Then any strong edge independent set in $G$ can be called as a strong matching. A maximum strong edge independent set is known as maximum strong matching.

Theorem 5.1. Let $G$ be a weighted graph. Let $M$ be a strong matching and let $S$ be a strong covering set of $G$. Then $|M| \leq|S|$.

Proof: Let $|M|=m$ and $|S|=s$. Let $e_{1}, e_{2}, \ldots e_{m}$ be the edges in strong matching. Then any strong covering set of $G$ should contain $m$ distinct vertices each of which are adjacent with these $m$ edges. Hence $m \leq s$.

Theorem 5.2. Let $G$ be a strongly bipartite weighted graph. Let $M$ be a maximum strong matching in $G$. Then $|M|=\alpha_{s}(G)$.

Proof: Let $V_{1}$ and $V_{2}$ be the partitions in which the end vertices of strong edges lie in $G$. Let $|M|=m$ and let $e_{1}, e_{2}, \ldots, e_{m}$ be the edges in $M$. Then any strong edge in $G$ will be adjacent to one end vertex of $e_{1}, e_{2}, \ldots, e_{m}$. Hence we can choose distinct vertices $v_{1}, v_{2}, \ldots, v_{m}$ in such a way that each strong edge in $G$ will be adjacent to either of these $m$ vertices. Hence $S=\left\{v_{1}, v_{2}, \ldots, v_{m}\right\}$ will form a strong covering set. $S$ is a minimum strong covering set as any other covering set $S^{\prime}$ with $\left|S^{\prime}\right|<|S|$ is a contradiction to above stated theorem. Hence $m=\alpha_{s}(G)$.

Remark 5.1. In a strongly bipartite weighted graph, its strong vertex/edge independence/covering number need not be equal to cardinalities of the partition sets in it. 


\section{Some Strong Connectivity Concepts in Weighted Graphs}

Consider the following example.

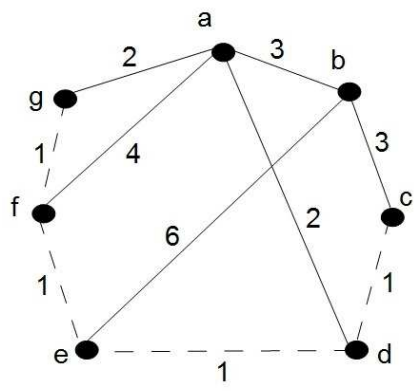

Figure 5: Weighted graph $G_{1}$

The above graph is a strongly bipartite weighted graph with the partition sets $V_{1}$ and $V_{2}$ (Figure 6), where $V_{1}=\{f, g, b, d\}$ and $V_{2}=\{a, c, e\}$.

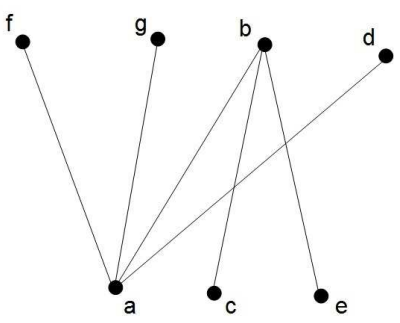

Figure 6: Strong edges in $G_{1}$

In $G_{1}$, the maximal strong independent set is $\{f, g, d, c, e\}$ and the minimal strong covering set is $\{a, b\}$. Hence $\alpha_{s}\left(G_{1}\right)=2$ and $\beta_{s}\left(G_{1}\right)=5$. Similarly one among the maximum strong edge independent sets is $\{(a, d),(b, c)\}$ and the minimum strong edge covering set is $\{(a, f),(a, g),(a, d),(b, c),(b, e)\}$. Hence $\alpha_{s}^{\prime}(G)=5$ and $\beta_{s}^{\prime}(G)=2$. We can see that none of them is equal to cardinality of any partition set.

\section{Conclusion}

In this paper, we have discussed the concepts of strong independent sets and strong covering sets of weighted graph. We have defined and studied strong independence number and strong covering number for different weighted graphs. Also we have found that the sum of strong independence number and strong covering number is a constant and is equal to the total number of vertices in a weighted graph. The edge analogues of the above concepts and results are also discussed in this paper. More properties and applications will be discussed in the forthcoming papers.

Acknowledgement. The authors are thankful to the referee for their valuable suggestions and helpful comments in writing this manuscript. We are grateful to National Institute of Technology Calicut for providing us with all the support required for this research paper.

\section{REFERENCES}




\section{Darshan Lal M Nair and Sunil Mathew}

1. J.A.Bondy, Hajo J. Broersma, Jan van den Heuvel and Henk Jan Veldman, Heavy cycles in weighted graphs, Discussiones Mathematicae Graph Theory, 22(1) (2002) $7-15$.

2. J.A.Bondy and G.Fan, Cycles in weighted graphs, Combinatorica, 11(3) (1991) 191-205.

3. J.A.Bondy and Genghua Fan, Optimal paths and cycles in weighted graphs, Ann. Discrete Mathematics, 41 (1989) 53-69.

4. Darshan Lal M Nair and Sunil Mathew, Some strong connectivity problems in weighted graphs, Journal of Uncertainty in Mathematics Science, 2014 (2014) 1-7.

5. R.Diestel, Graph theory, Springer-Verlag, 2000.

6. G.A.Dirac, Some theorems on abstract graphs, Proc. London Mathematics Society, 2(3) (1952) 69-81.

7. F.Harary, Graph Theory, Addison-Wesley, 1969.

8. M.Grotschel, Graphs with cycles containing given paths, Ann. Discrete Mathematics, 1 (1977) 233-245.

9. M.Pal, Intersection graphs: An introduction, Annals of Pure and Applied Mathematics, 4(1) (2013) 43-91.

10. S.Mathew, On cycle connectivity of graphs, Journal of Interconnection Networks, 13 (1-2) 1250005 (2012).

11. S.Mathew, Partial trees in weighted graphs-I, Proyecciones (Antofagasta), 30(2) (2011) 163-174.

12. S.Mathew and M.S.Sunitha, Some connectivity concepts in weighted graphs, Advances and Applications in Discrete Mathematics, 6(1) (2010) 45-54.

13. S.Mathew and M.S.Sunitha, A generalization of mengers theorem, Applied Mathematics Letters, 24(12) (2011) 2059-2063.

14. S.Mathew and M.S.Sunitha, A characterization of partial blocks in weighted graphs, Information Processing Letters, 112(17) (2012) 706-710.

15. S.Mathew and M.S.Sunitha, On totally weighted interconnection networks, Journal of Interconnection Networks, 1350004 (2013) 1-16.

16. M.S.Sunitha and S.Mathew, Fuzzy graph theory: A survey, Annals of Pure and Applied Mathematics, 4(1) (2013) 92-110.

17. D.K.Thakkar and A.A.Prajapati, Vertex covering and independence in semigraph, Annals of Pure and Applied Mathematics, 4(2) (2013) 172-181 